\title{
BROWNIAN MOTION WITH PARTIAL INFORMATION ${ }^{1}$
}

\author{
BY
}

TERRY R. MCCONNELL

\begin{abstract}
We study the following problem concerning stopped $N$-dimensional Brownian motion: Compute the maximal function of the process, ignoring those times when it is in some fixed region $R$. Suppose this modified maximal function belongs to $L^{q}$. For what regions $R$ can we conclude that the unrestricted maximal function belongs to $L^{q}$ ? A sufficient condition on $R$ is that there exist $p>q$ and a function $u$, harmonic in $R$, such that
\end{abstract}

$$
|x|^{p} \leqslant u(x) \leqslant C|x|^{p}+C, \quad x \in R
$$

for some constant $C$.

We give applications to analytic and harmonic functions, and to weak inequalities for exit times.

1. Introduction. Let $R$ be an open subset of $N$-dimensional Euclidean space $(N \geqslant 2)$. Suppose we start a Brownian motion, $B_{t}$, from some point outside $R$ and stop it at some finite exit time $T$, but we are unable to observe the motion whenever it is in $R$. (By exit time we mean exit time from some given open set $G$.) How much information about the entire Brownian path up to time $T$ is provided by this semiobscured path? We shall address a special case of this question here: Suppose the maximum Euclidean norm of the Brownian motion belongs to $L^{p}$ - the maximum being computed over that portion of its time set on which $B_{t}$ does not belong to $R$ and before time $T$. Does this imply that the unrestricted maximum (i.e., the maximum of $B_{t}$ up to time $T$ ) belongs to $L^{p}$ ? We shall see that the answer is yes (Theorem 1.1) provided $R$ is not too large.

We employ a technique of Burkholder, Gundy, and Silverstein [7] together with the conformal invariance of Brownian motion to translate the two-dimensional case of Theorem 1.1 into a statement about functions analytic in the unit disk.

We devote the remainder of this section to definitions and to the statements of our main results. These results are proved in $\$ 3$. In $\$ 2$ we consider several illustrative applications to classical analysis and probability. Finally, $\S 4$ is devoted to an example pertinent to some of the discussion below.

Received by the editors November 18, 1980.

1980 Mathematics Subject Classification. Primary 60J65; Secondary 60G46.

Key words and phrases. Exit time, Hardy space, Brownian motion, harmonic majorization, maximal function.

'This paper constitutes a portion of the author's Ph.D. dissertation written at the University of Illinois.

(C) 1982 American Mathematical Society 0002-9947/81/0000-0231/\$04.25 
Let $R$ be an open subset, not necessarily connected, of $\mathbf{R}^{N}$, and let $\tau$ denote the exit time of Brownian motion from $R$. We assume throughout that $N \geqslant 2$. Define the Brownian maximal function by

$$
B_{\tau}^{*}=\sup _{0 \leqslant t \leqslant \tau}\left|B_{t}\right| .
$$

We say that $R$ belongs to $H^{p}$ if one of the following three equivalent statements holds:

(1.1) For each $x$ in $R, E^{x} B_{\tau}^{* p}<\infty$.

(1.2) For each $x$ in $R, E^{x} \tau^{p / 2}<\infty$.

(1.3) The function $|x|^{p}$ has a harmonic majorant in $R$.

Both (1.1) and (1.2) hold provided the inequalities are true for some $x$ in each connected component of $R$. The equivalence of (1.1) and (1.2) follows from Theorem 7.1 of [6]; the equivalence of (1.3) with the other statements is proved in [4]. The reason for the terminology is that, in two dimensions, if $R$ is the image of the unit disk under a univalent analytic function $f$, then $R$ belongs to $H^{p}$ if and only if $f$ belongs to the Hardy space $H^{p}$. (See [4].)

We say that a region $R$ belongs to strong $H^{p}, 0<p<\infty$ (and write $R \in S H^{p}$ ), in case one of the following equivalent conditions holds for some constant $C$ :

$$
\begin{array}{ll}
E^{x} B_{\tau}^{* p} \leqslant C|x|^{p}+C, & x \in R . \\
E^{x} \tau^{p / 2} \leqslant C|x|^{p}+C, & x \in R .
\end{array}
$$

There is a function $u$, harmonic in $R$, so that

$$
|x|^{p} \leqslant u(x) \leqslant C|x|^{p}+C, \quad x \in R .
$$

The equivalence of (1.4) and (1.5) is an immediate consequence of the following inequality from $[4]$ :

$$
c_{p} E^{x}\left(N \tau+|x|^{2}\right)^{p / 2} \leqslant E^{x} B_{\tau}^{* p} \leqslant C_{p} E^{x}\left(N \tau+|x|^{2}\right)^{p / 2},
$$

where $c_{p}$ and $C_{p}$ are constants depending only on $p$. It is easy to see from (1.4) that $S H^{q} \subseteq S H^{p}$ for $q<p$. Any region $R$ which belongs to $H^{p}$ and is invariant under positive dilations (i.e., $\lambda x$ belongs to $R$ whenever $x$ belongs to $R$ and $\lambda>0$ ) also belongs to strong $H^{p}$; for example, in two dimensions a sector of angular operature $\theta$ belongs to strong $H^{p}$ for all $p<\pi / \theta$. (The classes $H^{p}$ and $S H^{p}$ are, however, quite different. In $\S 4$ we construct a region which belongs to $H^{p}$ for all positive $p$, but which belongs to $S H^{p}$ for no such p.) Symmetrization provides many further examples. Given an open connected subset $R$ of $\mathbf{R}^{N}$, define the (spherical) symmetrization, $R_{s}$, of $R$ as follows: Let $C(\theta)$ denote the set of points on the unit sphere which form an angle of less than $\theta$ with the positive $x_{1}$-axis. Let || denote normalized surface measure on $S^{N-1}$. Then $R_{s}$ is the region whose intersection with the sphere of radius $r$ is $r \cdot C(\theta)$, with $\theta$ determined so that

$$
|C(\theta)|=\left|\left\{x \in S^{N-1}: r x \in R\right\}\right| .
$$


If $\tau_{s}$ denotes the exit time of Brownian motion from $R_{s}$, then a theorem of Baernstein and Taylor [1, Corollary 6.2], suitably translated into probabilistic terms, says that

$$
\sup _{|x|=r} P^{x}\left(B_{\tau}^{*}>\lambda\right) \leqslant P^{(r, 0,0, \ldots, 0)}\left(B_{\tau_{s}}^{*}>\lambda\right),
$$

for all $\lambda>0$. The following result is then immediate.

Proposition 1.1. If $R_{s}$ belongs to $S H^{p}$, so does $R$.

Another criterion for membership in $S H^{p}$ may be found in [15, Theorem III.70].

Theorem 1.1 below tells what is meant by the phrase "not too large" in the first paragraph: In order to determine whether the maximal function, $B_{T}^{*}$, of Brownian motion up to some finite exit time $T$ belongs to $L^{q}$, we may ignore that portion of the path lying in some open set $R$, provided $R$ belongs to strong $H^{p}$ for some $p>q$. By exit time, we mean the first time Brownian motion leaves some given open set. For the remainder of this paper the letter $T$ will stand for such a finite exit time of Brownian motion. The letter $R$ will denote an open set in which Brownian motion is invisible, and $\tau$ will represent the exit time from $R$. Define the obscured maximal function, $B_{T \backslash R}^{*}$, by

$$
B_{T \backslash R}^{*}=\sup _{0 \leqslant t<T: B_{t} \notin R}\left|B_{t}\right|
$$

Then we have

THEOREM 1.1. Let $R$ be a region belonging to $S H^{p}$ and $x_{0}$ a point not in the closure of $R$. Then for any $q<p$ there is a constant $C$ so that, for any exit time $T$, finite almost surely, we have

$$
E^{x_{0}}\left(B_{T}^{*}\right)^{q} \leqslant C E^{x_{0}}\left(B_{T \backslash R}^{*}\right)^{q} .
$$

The constant $C$ does not depend on $T$. Conversely, if (1.7) holds for all such $T$, then $R$ belongs to $\mathrm{SH}^{q}$.

We leave open the question of whether or not (1.7) holds when $q=p$. This question is generally irrelevant in applications since the range of $p$ for which a given region belongs to $S H^{p}$ is often an open interval.

We should point out that (1.7) holds if $R$ satisfies the weaker condition

$$
\lambda^{p} P^{x}\left(B_{\tau}^{*}>\lambda\right) \leqslant C|x|^{p}+C, \quad \lambda>0,
$$

for some constant $C$. Also, if $x_{0}$ belongs to the closure of $R$, then (1.7) may not hold. Nevertheless, the implication

$$
B_{T \backslash R}^{*} \in L^{q} \Rightarrow B_{T}^{*} \in L^{q}
$$

remains true.

Our next two results enable us to apply Theorem 1.1 to analytic and harmonic functions. If $x \in \mathbf{C}$ and $r>0$, let $D(x, r)$ denote the disk with center $x$ and radius $r$. For $0<\alpha<1$ and $\theta \in[0,2 \pi)$ let $\Omega_{\alpha}(\theta)$ be the smallest open convex subset of $D(0,1)$ whose closure contains the point $e^{i \theta}$ and the circle of radius $\alpha$. If $f$ is analytic 
in $D(0,1)$ and $R$ is an open subset of $\mathbf{C}$, let

$$
R N_{\alpha} f(\theta)=\sup _{z \in \Omega_{\alpha}(\theta): f(z) \notin R}|f(z)| .
$$

Also define

$$
R f^{*}=\sup _{0 \leqslant t<\sigma: f\left(B_{t}\right) \notin R}\left|f\left(B_{t}\right)\right|,
$$

where $\sigma$ is the exit time of Brownian motion from $D(0,1)$. Finally, define

$$
f^{*}=\sup _{0 \leqslant t<\sigma}\left|f\left(B_{t}\right)\right|=\varnothing f^{*} .
$$

THEOREM 1.2. Assume that the region $R$ satisfies

(1.8) $D(0, r) \cap R \neq \varnothing \Rightarrow$ each component of $D(0, r) \cup R$ is simply connected,

for all $r>0$. For each $\alpha>0$ there exist constants $c_{\alpha}$ and $C_{\alpha}$, depending only on $\alpha$, so that, for every function $f$ analytic in $D(0,1)$ and satisfying $f(0)=0$, we have

$$
c_{\alpha} m\left(R N_{\alpha} f>\lambda\right) \leqslant P\left(R f^{*}>\lambda\right) \leqslant C_{\alpha} m\left(R N_{\alpha} f>\lambda\right),
$$

for all $\lambda>0$, where $m$ is normalized Lebesgue measure.

The proof of this theorem is very similar to that of the analogous result $(R=\varnothing)$ in [7]. It will be sketched in $§ 3$.

THEOREM 1.3. Let $R$ be a region belonging to $S H^{p}$ with 0 not in the closure of $R$, and $f$ a function analytic in $D(0,1)$ with $f(0)=0$. Then for any $q$ satisfying $0<q<p$ there is a constant $C$ so that

$$
E^{0}\left(f^{*}\right)^{q} \leqslant C E^{0}\left(R f^{*}\right)^{q}
$$

2. Applications. In this section we show how our results may be applied by giving new proofs of two known results; and we obtain a new theorem concerning weak inequalities for exit times. Throughout the remainder of the paper we shall use the letter $C$ to denote a constant-perinaps different from one usage to the next.

THEOREM 2.1 [7]. Let $f=u+i v$ be analytic in $D(0,1)$ with $f(0)=0$, and let $\alpha$ and $p$ be numbers such that $0<\alpha<1$ and $0<p<\infty$. Then there exist constants $c$ and $C$ depending only on $\alpha$ and $p$ so that

$$
c\left\|N_{\alpha} u\right\|_{p} \leqslant\left\|N_{\alpha} v\right\|_{p} \leqslant C\left\|N_{\alpha} u\right\|_{p} .
$$

Here

$$
N_{\alpha} u(\theta)=\sup _{z \in \Omega_{\alpha}(\theta)}|u(z)| .
$$

This result easily implies [7] that $f \in H^{p} \Leftrightarrow N_{\alpha} u \in L^{p}$, yielding a real variable characterization of the Hardy space $H^{p}$. The authors of [7] give two proofs of this result, one of them using the quadratic variation,

$$
S^{2}(u)=\int_{0}^{\sigma}\left|\nabla u\left(B_{s}\right)\right|^{2} d s
$$

as a bridge between $u$ and $v$. In our proof the function $f$ itself plays this role. 
Proof of Theorem 2.1. We shall use the notation $\left\|N_{\alpha} u\right\|_{p} \approx\left\|N_{\alpha} v\right\|_{p}$ to indicate a two-sided inequality such as (2.1). We may assume that $D(0,1) \subseteq f\left(D\left(0, \frac{1}{2}\right)\right)$ by the open mapping theorem and the invariance of (2.1) under scaling. Also, we may assume that $\alpha=\frac{1}{2}$ (see [13]) and $0<p<2$. (For $p>1$ we have $\left\|N_{\alpha} u\right\|_{p} \approx\|u\|_{h^{p}}$. See, e.g., [14, Theorem 7.1]. Clearly $\|u\|_{h^{2}}=\|v\|_{h^{2}}$ and $\|u\|_{h^{p}} \approx\|v\|_{h^{p}}$ for $p>2$ by duality. Recall that $\|u\|_{h^{p}}$ is defined by

$$
\left.\|u\|_{h^{p}}^{p}=\sup _{0<r<1} \int_{0}^{2 \pi}\left|u\left(r e^{i \theta}\right)\right|^{p} d \theta .\right)
$$

Let $R=\left\{z=x+i y:|y|>|x|\right.$ and $\left.|z|>\frac{1}{2}\right\}$. Then $R$ belongs to $S H^{p}$ for all $0<p<2$. By Theorems 1.2 and 1.3, we have

$$
\begin{aligned}
\left\|N_{\alpha} v\right\|_{p} & \leqslant\left\|N_{\alpha} f\right\|_{p} \leqslant C\left\|f^{*}\right\|_{p} \leqslant C\left\|R f^{*}\right\|_{p} \\
& \leqslant C\left\|R N_{\alpha} f\right\|_{p} \leqslant C\left\|N_{\alpha} u\right\|_{p} .
\end{aligned}
$$

Similarly one proves that $\left\|N_{\alpha} u\right\|_{p} \leqslant C\left\|N_{\alpha} v\right\|_{p}$.

TheOREM 2.2 [3]. Let $u$ be harmonic in $D(0,1)$ with $u(0)=0$, and let $\alpha$ and $p$ be numbers satisfying $0<\alpha<1$ and $0<p<\frac{2}{3}$. Then there is a constant $C$, depending only on $\alpha$ and $p$, such that

$$
\left\|N_{\alpha} u\right\|_{p} \leqslant C\left\|N_{\alpha} u^{+}\right\|_{p}
$$

where $u^{+}=u \vee 0$.

This result is also true in higher dimensions and for all $p$ satisfying $0<p<1$ [3], but our methods give only this partial result.

Proof of TheOrem 2.2. Let $v$ be the harmonic function conjugate to $u$ with $v(0)=0$, and let $f=u+i v$. As in the proof of Theorem 2.1 we may assume that $\alpha=\frac{1}{2}$ and that $f\left(D\left(0, \frac{1}{2}\right)\right)$ contains $D(0,1)$. Arguing as in the proof of Theorem 2.1, we obtain

$$
\left\|N_{\alpha}(v I\{u>0\})\right\|_{p} \leqslant C\left\|N_{\alpha} u^{+}\right\|_{p}
$$

for $0<p<\frac{2}{3}$, where $I\{u>0\}$ is the indicator function of the set where $u$ is positive. (Modify the set $R$ in the proof of Theorem 2.1 by adjoining the set $\{z=x+i y: x<0$ and $\left.|z|>\frac{1}{2}\right\}$.) Now let $R=\left\{z=x+i y: x<0,|z|>\frac{1}{2}\right\}$. Then

$$
\left\|N_{\alpha} u\right\|_{p} \leqslant C\left\|R N_{\alpha} f\right\|_{p} \leqslant C\left\|N_{\alpha} u^{+}\right\|_{p}+C\left\|N_{\alpha}(v I\{u>0\})\right\|_{p} \leqslant C\left\|N_{\alpha} u^{+}\right\|_{p},
$$

and the proof is complete.

The following result is an immediate consequence of Theorems 1.2 and 1.3.

THEOREM 2.3. Let $R$ be a sector of angular aperture $\theta$ with vertex at the origin, and let $p$ satisfy $0<p<\pi / \theta$. If $f$ is analytic in $D(0,1)$ then

$$
f \in H^{p} \Leftrightarrow R N_{\alpha} f \in L^{p}
$$

for any $0<\alpha<1$.

Let us turn to another sort of application. If $Y$ is a random variable defined on a probability space we say that $Y$ belongs to weak $L^{p}$, or $Y \in W L^{p}$, if there is a 
constant $c$ so that we have

$$
\lambda^{p} P(|Y|>\lambda) \leqslant c, \quad \lambda>0 .
$$

Let $G$ be a connected open subset of $\mathbf{R}^{N}$ and $T$ the exit time of Brownian motion from $G$. Assume that $P^{x}(T<\infty)=1$ for some $x$ in $G$. Then it is known [5] that

$$
B_{T}^{*} \in W L^{p} \Leftrightarrow T^{1 / 2} \in W L^{p} .
$$

We shall say that $G$ belongs to weak $H^{p}\left(G \in W H^{p}\right)$ in case $B_{T}^{*} \in W L^{p}$. It is natural to ask for a nonprobabilistic characterization of $W H^{p}$ similar to the characterization (1.3) of $H^{p}$. The following theorem provides a useful sufficient condition for $W H^{p}$.

THEOREM 2.4. For $u$ harmonic in $G$ and $p>0$ let

$$
w_{s}(r)=\left|\left\{x \in S^{N-1}: u(r x) \leqslant s r^{p}, r x \in G\right\}\right| .
$$

If there exists a harmonic function $u$ for which

$$
\lim _{s \rightarrow 0} \limsup _{r \rightarrow \infty} w_{s}(r)=0,
$$

then $G \in W H^{p}$.

For example, if $G$ is the sector in $\mathbf{C}$ defined by $G=\left\{r e^{i \theta}: 0<\theta<\theta_{0}\right\}$ then $G \in W H^{p}$ when $p=\pi / \theta_{0}$. (However, $G \notin H^{p}$. See [4] for this and the analogous results for cones in higher dimensions.) We may take here $u\left(r e^{i \theta}\right)=r^{p} \sin p \theta$. The fact that $G$ belongs to $W H^{p}$ is shown by a different method in [5].

We will prove Theorem 2.4 in $\$ 3$.

3. Proofs of the theorems. To prove norm inequalities, we shall first prove distribution function inequalities as in the work of Burkholder and Gundy [6]. We shall use the following special case of [2, Lemma 7.1].

LEMMA 3.1. Let $f$ and $g$ be nonnegative functions on a probability space and $\beta, \delta$ and $\varepsilon$ positive numbers satisfying $\beta>1, \delta>0$ and

$$
P(f>\beta \lambda, g \leqslant \delta \lambda)<\varepsilon P(f>\lambda), \quad \lambda>0 .
$$

Let $q$ satisfy $0<q<\infty$ and $\beta^{q} \varepsilon<1$. Then we have [2 and 5 ]

$$
E f^{q} \leqslant c E g^{q}
$$

and

$$
\sup _{\lambda>0} \lambda^{q} P(f>\lambda) \leqslant c \sup _{\lambda>0} \lambda^{q} P(g>\lambda),
$$

where $c=\left[\beta^{q} / \delta^{q}\left(1-\beta^{q} \varepsilon\right)\right]$.

Proof of Theorem 1.1. Assume for the time being that $N=2$ and $x_{0}=0$, and fix a positive number $\rho$ such that $D(0, \rho)$ does not meet $R$. Let $\beta, \delta$, and $\lambda$ be positive real numbers as in Lemma 3.1 with $\delta<1$. We first prove the following distribution function inequality:

$$
P^{0}\left(B_{T}^{*}>\beta \lambda, B_{T \backslash R}^{*} \leqslant \delta \lambda\right) \leqslant \varepsilon(\beta, \delta) P^{0}\left(B_{T}^{*}>\lambda\right),
$$


where $\varepsilon(\beta, \delta)=c\left(\beta^{-p}+\log \beta / \log \beta \delta^{-1}\right)$. Here $c$ is a constant depending only on $p$ and $\rho$. Letting $\mu(\lambda)=\inf \left\{t:\left|B_{t}\right|=\lambda\right\}$, we have by the strong Markov property,

$$
\begin{aligned}
P^{0}\left(B_{T}^{*}>\right. & \left.\beta \lambda, B_{T \backslash R}^{*} \leqslant \delta \lambda\right)=P^{0}\left(B_{T}^{*}>\beta \lambda, B_{T \backslash R}^{*} \leqslant \delta \lambda, \mu(\lambda)<T, B_{\mu(\lambda)} \in R\right) \\
\leqslant & E^{0}\left(P^{B_{\mu(\lambda)}}\left(B_{T}^{*}>\beta \lambda, B_{T \backslash R}^{*} \leqslant \delta \lambda\right) ; \mu(\lambda)<T, B_{\mu(\lambda)} \in R\right) \\
\leqslant & E^{0}\left(P^{B_{\mu(\lambda)}}\left(B_{\tau}^{*}>\beta \lambda\right) ; \mu(\lambda)<T, B_{\mu(\lambda)} \in R\right) \\
& +E^{0}\left(P^{B_{\mu(\lambda)}}\left(B_{t} \text { hits the circle of radius } \delta \lambda \text { before the circle of radius } \beta \lambda\right) ;\right. \\
= & I+I I .
\end{aligned}
$$

By the Hadamard Three Circles Theorem,

$$
\begin{aligned}
I I & \leqslant\left(\log \beta / \log \beta \delta^{-1}\right) P^{0}(\mu(\lambda)<T) \\
& =\left(\log \beta / \log \beta \delta^{-1}\right) P^{0}\left(B_{T}^{*}>\lambda\right) .
\end{aligned}
$$

Using Chebyshev's inequality and (1.4) we have

$$
\begin{aligned}
P^{B_{\mu(\lambda)}}\left(B_{\tau}^{*}>\beta \lambda\right) & \leqslant \frac{1}{\lambda^{p} \beta^{p}} E^{B_{\mu(\lambda)}}\left(B_{\tau}^{* p}\right) \\
& \leqslant \frac{1}{\lambda^{p} \beta^{p}}\left(C\left|B_{\mu(\lambda)}\right|^{p}+C\right)=\frac{C}{\beta^{p}}+\frac{C}{\beta^{p} \lambda^{p}} \quad \text { on }\left\{B_{\mu(\lambda)} \in R\right\} .
\end{aligned}
$$

Hence

$$
I \leqslant\left(C \beta^{-p}+C \beta^{-p} \lambda^{-p}\right) P^{0}\left(\mu(\lambda)<T, B_{\mu(\lambda)} \in R\right) .
$$

Since $\{\mu(\lambda)<T\}=\left\{B_{T}^{*}>\lambda\right\}$, we have

$$
I \leqslant C \beta^{-p} P^{0}\left(B_{T}^{*}>\lambda\right)+C \beta^{-p} \lambda^{-p} P^{0}\left(B_{T}^{*}>\lambda, B_{\mu(\lambda)} \in R\right) .
$$

The second term imposes the restriction that $\lambda>\rho$, hence

$$
I \leqslant C \beta^{-p} P^{0}\left(B_{T}^{*}>\lambda\right),
$$

where $C$ depends on $p$ and $\rho$. Adding together the estimates for $I$ and $I I$ gives (3.3). Now, since $q<p$, we may first take $\beta$ very large and then $\delta$ very small so that $\beta^{q} \varepsilon(\beta, \delta)<1$. Lemma 3.1 then implies that

$$
E^{0}\left(B_{T}^{*}\right)^{q} \leqslant C E^{0}\left(B_{T \backslash R}^{*}\right)^{q} .
$$

Suppose now that $x_{0} \neq 0$. Let $T(G)$ denote the exit time of $B_{t}$ from some open set $G$ containing $x_{0}$. Then we have

$$
E^{x_{0}}\left(B_{T(G)}^{*}\right)^{q} \leqslant C\left[E^{0}\left(B_{T\left(G-x_{0}\right)}^{*}\right)^{q}+\left|x_{0}\right|^{q}\right]
$$

where $G-x_{0}$ denotes the translation of $G$ by $x_{0}$. Now $R \in S H^{p} \Rightarrow R-x_{0} \in S H^{p}$ with new constants depending on $x_{0}$ in (1.4)-(1.6). Moreover, we have

$$
E^{0}\left(B_{T\left(G-x_{0}\right) \backslash\left(R-x_{0}\right)}^{*}\right)^{q} \leqslant C\left[E^{x_{0}}\left(B_{T(G) \backslash R}^{*}\right)^{q}+\left|x_{0}\right|^{q}\right] .
$$


Inequality (1.7) with $T=T(G)$ follows from the two preceding inequalities and (3.5) with $T=T\left(G-x_{0}\right)$ and $R$ replaced by $R-x_{0}$. Note that $x_{0}$ not in the closure of $R$ implies $\left|x_{0}\right|^{q} \leqslant E^{x_{0}}\left(\left|B_{T \backslash R}^{*}\right|^{q}\right)$.

The proof in the case $N>2$ is the same, but with the constant in (3.4) replaced by $\left(1-\beta^{2-N}\right) /\left(\delta^{2-N}-\beta^{2-N}\right)$.

As to the converse, we shall again assume at first that $N=2$. Let $C_{r}=\partial D\left(x_{0}, r\right)$ for each $r>0$ and let $m$ denote normalized Lebesgue measure on $C_{r}$. Applying the strong Markov property and (1.7) with $T$ the exit time from the region $R \cup D\left(x_{0}, r\right)$, we obtain

$$
\begin{aligned}
C r^{q} & \geqslant E^{x_{0}}\left(B_{T}^{* q}\right) \geqslant E^{x_{0}}\left(E^{B_{\mu(r)}}\left(B_{\tau}^{* q}\right) ; B_{\mu(r)} \in R\right) \\
& =\int_{C_{r} \cap R} E^{x}\left(B_{\tau}^{* q}\right) m(d x),
\end{aligned}
$$

where $\mu(r)=\inf \left\{t:\left|B_{t}-x_{0}\right|=r\right\}$. In the last step we used Kakutani's observation that if a Brownian motion is started from $x_{0}$, then its position upon exit from $D\left(x_{0}, r\right)$ is uniformly distributed on $C_{r}$.

We may assume $R \neq \varnothing$. Since each component of $R$ is met in a set of positive measure by $C_{r}$ for some $r$, it follows that $E^{x} B_{\tau}^{* q}<\infty$ for some $x$ in each component of $R$. Therefore $|x|^{q}$ has a harmonic majorant on $R$, and the least such majorant, $u$, is given by $u(x)=E^{x}\left|B_{\tau}\right|^{q}$. It follows that

$$
\int_{C_{r} \cap R} u(x) m(d x) \leqslant C r^{q}, \quad r>0 .
$$

Given a number $\lambda \geqslant 1$, let $R^{\lambda}=\left\{x \in R: u(x)>\lambda|x|^{q}\right\}$. If $R^{\lambda}$ is connected we may proceed as follows (to handle the other case simply apply the following argument to each component in turn). Let $T_{\lambda}$ be the exit time of Brownian motion from $R^{\lambda}$. By Chebyshev's inequality and (3.6) we have

$$
\lambda m\left\{x \in C_{r} \cap R: u(x)>\lambda r^{q}\right\} \leqslant C .
$$

Choose $\lambda$ so large that $C / \lambda<1 / 2 q$. Then the circular symmetrization (defined in the usual way but with $x_{0}$ as origin) of $R^{\lambda}$ is contained in a sector of angular aperture less than $\pi / q$. Such a sector satisfies (1.6) and, hence, so does $R^{\lambda}$ by virtue of Proposition 1.1. Thus there exists a harmonic function, $v$, which majorizes $|x|^{q}$ on $R^{\lambda}$ and satisfies $v(x) \leqslant C|x|^{q}+C$. (In the disconnected case the same $C$ will work for each component.)

Now, for $x$ in $R^{\lambda}$, we have

$$
\begin{aligned}
u(x) & =E^{x}\left(\left|B_{\tau}\right|^{q}\right)=E^{x}\left(\left|B_{T_{\lambda}}\right|^{q} ; T_{\lambda}=\tau\right)+E^{x}\left(\left|B_{\tau}\right|^{q} ; T_{\lambda}<\tau\right) \\
& \leqslant v(x)+E^{x}\left(E^{B_{T_{\lambda}}}\left(\left|B_{\tau}\right|^{q}\right) ; T_{\lambda}<\tau\right) \\
& =v(x)+E^{x}\left(u\left(B_{T_{\lambda}}\right) ; T_{\lambda}<\tau\right) \\
& \leqslant v(x)+\lambda E^{x}\left(\left|B_{T_{\lambda}}\right|^{q} ; T_{\lambda}<\tau\right) \\
& \leqslant v(x)+\lambda v(x) \leqslant C(1+\lambda)|x|^{q}+C(1+\lambda) .
\end{aligned}
$$

Since $u(x) \leqslant \lambda|x|^{q}$ for $x \notin R^{\lambda}$, it follows that $u$ satisfies (1.6) with $p$ replaced by $q$. Hence $R \in S H^{q}$. 
The proof of the $N$-dimensional case is very similar: Given $\theta>0$, let $S_{\theta}$ denote the cone of all vectors in $\mathbf{R}^{N}$ making an angle of less than $\theta$ with the positive $x_{1}$-axis. Then, for each $p$, there is $\theta_{0}=\theta_{0}(p)$ sufficiently small so that $S_{\theta} \in S H^{p}$ for $\theta<\theta_{0}$ [4]. These cones, translated so as to have vertex at $x_{0}$, replace the two-dimensional sectors in the argument above. Otherwise the argument is the same.

Proof OF THEOREM 1.2. We shall only sketch this proof since it is very similar to that of [7, Theorem 3] (the case $R=\varnothing$ ) as presented in [13]. The proof of the right-hand side inequality of Theorem 1.2 uses only the lower semicontinuity of $R N_{\alpha} f$ and certain properties of Brownian motion. The proof is the same as the $R=\varnothing$ case.

For the left-hand side, begin by defining

$$
R M_{\alpha} f(\theta)=\sup _{z \in \Omega_{\alpha}(\theta):|z|>\alpha, f(z) \notin R}|f(z)| .
$$

Then it suffices to prove $C_{\alpha} m\left(R M_{\alpha} f>\lambda\right) \leqslant P\left(R f^{*}>\lambda\right)$ (see [13]).

Let $P_{\theta}^{z}$ be the Wiener measure associated with Brownian motion started from the point $z$ and conditioned to hit the unit circle at the point $e^{i \theta}$. The following facts about $P_{\theta}^{z}$ are known [9].

(i) Brownian motion forms a strong Markov proccess under the $P_{\theta}^{z}$.

(ii) The family $P_{\theta}^{z}$ yields a regular version of the conditional probabilities $P^{z}\left(\cdot \mid B_{\sigma}\right)$. (Recall that $\sigma$ is the exit time of $B_{t}$ from the unit disk.)

(iii) If $T$ is any stopping time then

$$
P_{\theta}^{z}(T<\sigma)=\frac{1}{h(z)} E^{z}\left(h\left(B_{T}\right) ; T<\sigma\right),
$$

where $h$ is the minimal harmonic function (Poisson kernel) with a pole at $e^{i \theta}$.

Suppose $\lambda$ is given and $\theta$ is such that $R M_{\alpha} f(\theta)>\lambda$. Define $\hat{B}_{t}$ by $\hat{B}_{t}=e^{2 i \theta} \bar{B}_{t}$ and

$$
R^{*} f=\sup _{0 \leqslant t<\sigma: f\left(\hat{B}_{t}\right) \notin R}\left|f\left(\hat{B}_{t}\right)\right| .
$$

The process $\hat{B}_{t}$ is simply Brownian motion reflected across the line joining 0 and $e^{i \theta}$. Probabilistically it is again a Brownian motion. Given $r$ with $\alpha<r<1$, let $a_{r}$ be the intersection of the circle of radius $\frac{1}{2}(1+r)$, tangent to the unit circle at $-e^{i \theta}$, with $\Omega_{\alpha}(\theta)$. We may choose such an $r$ so that, for some point $z$ on $a_{r}$, we have $|f(z)|>\lambda$ and $f(z) \notin R$. We shall prove that, apart from a set of $\omega$ of $P_{\theta}^{0}$-probability 0 , if $B_{t}(\omega)$ does not hit $a_{r}$ then $R^{*} f(\omega) \vee R f^{*}(\omega)>\lambda$. (Once this key fact has been established, the remainder of the proof proceeds as in the case $R=\varnothing$.) This fact, as we shall show, follows from the following two lemmas. The first serves as an analogue of the maximum modulus principle and is an easy consequence of the argument principle.

LemMa 3.2. Let $G$ and $W$ be simply connected regions with $G$ bounded. Let $f$ be analytic in $G$ and continuous on $\bar{G}$ with $f(\partial G) \subseteq W$. Then $f(G) \subseteq W$.

LEMMA 3.3. For any $\varepsilon>0$,

$$
P_{0}^{0}\left(B_{t} \text { hits }[1-\varepsilon, 1) \text { infinitely often }\right)=1 .
$$


Proof. We shall prove an analogous result in the upper half-plane-this is done only for notational convenience and ease of computation. Let $P_{0}^{z}$ denote the Wiener measure associated with Brownian motion started at a point $z$ in the upper half-plane and conditioned to hit the $x$ axis at the point $(0,0)$. We shall show that $B_{t}$ hits every segment $(0, \varepsilon i], \varepsilon>0$, infinitely often with $P_{0}^{z}$-probability 1 .

Let $r>0$ be fixed, $S$ be the segment ( $r i / 4, r i / 2$ ], $\Gamma$ the semicircle of radius $r$ centered at 0 , and $T_{S}$ the hitting time of $B_{t}$ to $S$. We first show that there is an absolute constant $C$ so that

$$
P_{0}^{z}\left(B_{t} \text { hits } S\right) \geqslant C,
$$

for $|z|>r$. Since the conditioned motion must eventually hit $\Gamma$, it follows from the strong Markov property that we need only consider $z \in \Gamma$; and, by symmetry, we may restrict our attention to $z$ belonging to the first quadrant. Let $h(x+i y)=$ $y /\left(\pi\left(x^{2}+y^{2}\right)\right)$ be the Poisson kernel with a pole at 0 . Then

$$
P_{0}^{z}\left(B_{t} \text { hits } S\right)=P_{0}^{z}\left(T_{S}<\mathfrak{e}\right)=\frac{1}{h(z)} E^{z}\left(h\left(B_{T_{S}}\right) ; T_{S}<\varrho\right),
$$

where $\mathcal{E}$ denotes the exit time of Brownian motion from the upper half-plane. To estimate this last quantity we distinguish two cases.

Case I. $\operatorname{Re}(z) \leqslant \operatorname{Im}(z)$.

We have $h(z) \leqslant 1 /(\pi r)$ and $h\left(B_{T_{S}}\right) \geqslant 2 /(\pi r)$ so

$$
P_{0}^{z}\left(B_{t} \text { hits } S\right) \geqslant 2 P^{z}\left(T_{S}<\mathcal{\varrho}\right)=2 \omega(z ; S),
$$

where $\omega(z ; S)$ denotes the harmonic measure at $z$ of $S$ relative to the upper half-plane. By the scaling properties of Brownian motion, this harmonic measure is independent of $r$. It follows that $\omega(z ; S)$ is bounded away from 0 by a positive absolute constant.

Case II. $\operatorname{Re}(z)>\operatorname{Im}(z)$.

With the notation as above we obtain

$$
P_{0}^{z}\left(B_{t} \text { hits } S\right) \geqslant \frac{2 r}{y} \omega(z ; S),
$$

where $y=\operatorname{Im}(z)$. An easy estimate shows that

$$
\omega(z ; S)>\frac{6}{25 \pi} \frac{x y}{r^{2}} .
$$

(This estimate is expedited by the observation that

$$
\omega(z ; S)>\omega\left(z^{2} ;\left[-r^{2} / 4,-r^{2} / 16\right)\right),
$$

which in turn follows from the fact that $\omega(z ; S)$ is greater than the harmonic measure of $S$ relative to the first quadrant.) Since $x \geqslant \sqrt{2} r / 2$ in this case, we obtain $\omega(z ; S) \geqslant C y / r$, whence

$$
P_{0}^{z}\left(B_{t} \text { hits } S\right) \geqslant \frac{2 r}{y} \cdot c \frac{y}{r}=C
$$

Thus (3.7) is proved.

Now let $A_{n}$ be the event that $B_{t}$ hits the interval $\left(2^{-2 n} \varepsilon i, 2^{-2 n+1} \varepsilon i\right]$. Then $P_{0}^{z}\left(A_{n}\right) \geqslant C$ so that $P_{0}^{z}\left(B_{t}\right.$ hits $(0, i \varepsilon]$ infinitely often $) \geqslant P_{0}^{z}\left(A_{n}\right.$ i.o. $)>0$. Since $\lim _{t \rightarrow E^{-}} B_{t}=0$ 
$P_{0}^{z}$-almost surely [9], the result follows from an application of the Blumenthal zero-one law to the time reversed process (see [12]).

Let $A$ be the exceptional set in Lemma 3.3. If $\omega \notin A$ and the path $B_{t}(\omega)$ avoids $a_{r}$, then the union of the paths $B_{t}(\omega)$ and $\hat{B}_{t}(\omega)$ contains a closed curve $\gamma$ which surrounds $a_{r}$, i.e., $a_{r}$ is contained in some bounded component of the complement of $\gamma$. Call this component $G$ and observe that it is simply connected. If $D(0 ; \lambda)$ meets $R$, let $V=R \cup D(0, \lambda)$. Otherwise, set $V=D(0, \lambda)$. Then by hypothesis, each component of $V$ is simply connected. Suppose it were false that $R f^{*}(\omega) \vee R^{*} f(\omega)>$ $\lambda$. Then the image of $\partial G$ under $f$ is contained in some component $W$ of $V$. But then Lemma 3.1 implies that $f(G) \subseteq W$, hence $f\left(a_{r}\right) \subseteq W$, a contradiction.

Proof of TheOrem 1.3. First recall Levy's observation of the conformal invariance of Brownian motion. There is a new Brownian motion, $W$, on the range of $f$ such that $f\left(B_{t}\right)=W_{\nu(t)}, t<\sigma$, where $\nu(t)=\int_{0}^{t}\left|f^{\prime}\left(B_{s}\right)\right|^{2} d s$.

Fix $\lambda, \beta$, and $\delta$ as in (3.3) and let $\mu=\inf \left\{t:\left|f\left(B_{t}\right)\right|=\lambda\right\}$. Using the strong Markov property as in the proof of (3.3), we have

$$
P^{0}\left(f^{*}>\beta \lambda, R f^{*} \leqslant \delta \lambda\right) \leqslant E^{0}\left(\Phi\left(B_{\mu}\right) ; \mu<\sigma, f\left(B_{\mu}\right) \in R\right),
$$

where $\Phi(x)=P^{x}\left(f^{*}>\beta \lambda, R f^{*} \leqslant \delta \lambda\right)$, for $f(x) \in R$. By Levy's observation we have

$$
\Phi(x)=P^{f(x)}\left(W_{\nu(\sigma)}^{*}>\beta \lambda, R W_{\nu(\sigma)}^{*} \leqslant \delta \lambda\right) .
$$

If $x$ satisfies $f(x) \in R$ and $|f(x)|=\lambda$, the last expression is dominated by $P^{f(x)}\left(W_{\tau}^{*}>\beta \lambda\right)+P^{f(x)}$ (the Brownian motion $W_{t}$ hits the circle of radius $\delta \lambda$ before that of radius $\beta \lambda$ ), where $\tau$ is the exit time of $W_{t}$ from $R$. Proceeding as in the proof of (3.3) we deduce the distribution function inequality

$$
P^{0}\left(f^{*}>\beta \lambda, R f^{*} \leqslant \delta \lambda\right) \leqslant \varepsilon(\beta, \delta) P^{0}\left(f^{*}>\lambda\right),
$$

where $\varepsilon(\beta, \delta)$ is the same as in (3.3). The remainder of the proof is exactly the same as the proof of Theorem 1.1.

Proof of Theorem 2.4. Let $p^{\prime}$ satisfy $p^{\prime}>p$. Suppose there is a function $u$ harmonic in $G$ for which $\lim _{s \rightarrow 0} \lim \sup _{r \rightarrow \infty} w_{s}(r)=0$. We may therefore find $r$ and $s$ so that the symmetrization of the region

$$
R=\left\{x \in G:|x|>r \text { and } u(x)<s|x|^{p}\right\}
$$

is contained in a very thin cone. (See the discussion at the end of the proof of Theorem 1.1.) Thus Proposition 1.1 implies that we may take $r$ and $s$ so that $R$, as defined above, belongs to $S H^{p^{\prime}}$. By (3.3) (and its $N$-dimensional version) and (3.2) it suffices to show that $\lambda^{p} P^{x}\left(B_{T \backslash R}^{*}>\lambda\right)=O(1)$ as $\lambda \rightarrow \infty$, for any $x \in G$. Here $T$ is the exit time of $B_{t}$ from $G$. Let $G_{n} \uparrow G$ be an exhaustion of $G$ by open sets whose closures are compact, and let $T(n)$ be the exit time of $B_{t}$ from $G_{n}$. It follows from Doob's maximal inequality that, for $\lambda>r^{p}$, we have

$$
\begin{aligned}
\lambda P^{x}\left(\left|R B_{T(n)}^{*}\right|^{p}>\lambda\right) & \leqslant \lambda P^{x}\left(\sup _{0 \leqslant t<T(n)} u\left(B_{t}\right) \geqslant s \lambda\right) \\
& \leqslant \frac{1}{s} E^{x} u\left(B_{T(n)}\right)=u(x) / s .
\end{aligned}
$$

The result follows upon passing to the limit as $n \rightarrow \infty$. 
4. An example. In this section we construct an example to illustrate the difference between $H^{p}$ and $S H^{p}$.

THEOREM 4.1. There exists a region $R$ belonging to $H^{p}$ for every positive $p$ and belonging to no such $\mathrm{SH}^{p}$.

We shall need the following lemma which follows easily from the three circles theorem.

LEMMA 4.1. Let $\tau_{r R}$ be the exit time of Brownian motion from the annulus with center 0 , small radius $r$, and large radius $R$. Then $\lim _{R \rightarrow \infty} E^{2 r}\left(\tau_{r R}^{p}\right)=\infty$, for any $p>0$.

Proof of Theorem 4.1. Let $\left\{p_{n}\right\}$ be an ordering of the rationals in $(0, \infty)$. Construct inductively an increasing sequence, $\left\{r_{n}\right\}$, of positive real numbers by setting $r_{0}=1$ and requiring that

$$
E^{2 r_{n-1}}\left(\tau_{r_{n-1} r_{n}}^{p_{k}}\right)>n^{p_{k}}\left(2 r_{n-1}\right)^{2 p_{k}},
$$

for $k=1,2, \ldots, n$. Lemma 4.1 ensures that this is possible. Let $D_{n}$ be the circle of radius $r_{n}$ minus the arc $\left\{r_{n} e^{i \theta}:|\theta|<\varepsilon_{n}\right\}$, where the $\varepsilon_{n}$ will be fixed shortly. Let $R=\left(\cup_{n=1}^{\infty} D_{n}\right)^{c}$. We will use the notation $\left[-\varepsilon_{n}, \varepsilon_{n}\right]$ to refer to the $\operatorname{arc}\left\{r_{n} e^{i \theta}:|\theta|<\right.$ $\left.\varepsilon_{n}\right\}$. Let $\tau_{n}$ be the exit time of $B_{t}$ from $D\left(0, r_{n}\right)$, and

$$
\mathbb{Q}_{n}=\left\{\tau_{i}<\tau, i=1,2, \ldots, n-1, \tau_{n}=\tau\right\}
$$

(Recall that $\tau$ is the exit time of $B_{t}$ from $R$.) By the strong Markov property

$$
\begin{aligned}
P^{0}\left(Q_{n}\right) & \leqslant E^{0}\left(P^{B_{\tau_{n-2}}}\left(B_{\tau_{n-1}} \in\left[-\varepsilon_{n-1}, \varepsilon_{n-1}\right]\right)\right) \\
& \leqslant P^{r_{n-2}}\left(B_{\tau_{n-1}} \in\left[-\varepsilon_{n-1}, \varepsilon_{n-1}\right]\right),
\end{aligned}
$$

for $n \geqslant 3$. This quantity approaches 0 as $\varepsilon_{n-1}$ approaches 0 . Thus we may choose $\varepsilon_{n}$ so that

$$
E^{0}\left(\tau_{n}^{p_{k}}\right)^{1 / 2} P^{0}\left(Q_{n}\right)^{1 / 2} \leqslant 2^{-n}
$$

for $k=1,2, \ldots, n$.

Now the region $R$ has been completely specified and it remains to show that it has the required properties. Fix $N>0$ and $p \in(0, \infty)$, and choose $n$ so large that $n^{p}>N$ and so that the set $\left\{p_{1}, \ldots, p_{n}\right\}$ contains some $p_{k}$ in the interval $(0, p)$. It follows from (4.1) that

$$
E^{2 r_{n-1}}\left(\tau^{p}\right)^{1 / p} \geqslant E^{2 r_{n}-1}\left(\tau_{r_{n-1} r_{n}}^{p}\right)^{1 / p} \geqslant E^{2 r_{n} \cdot 1}\left(\tau_{r_{n}-r_{n} r_{n}}^{p_{k}}\right)^{1 / p_{k}}>n\left(2 r_{n-1}\right)^{2} .
$$

Thus $E^{2 r_{n-1}}\left(\tau^{p}\right)>N\left(2 r_{n-1}\right)^{2 p}$. Since $N$ was arbitrary, $R \notin S H^{2 p}$. (Statement (1.5) fails to hold.)

Using the Schwartz inequality we have

$$
E^{0}\left(\tau^{p / 2}\right)=\sum_{n=1}^{\infty} E^{0}\left(\tau_{n}^{p / 2}, Q_{n}\right) \leqslant \sum_{n=1}^{\infty} E^{0}\left(\tau_{n}^{p}\right)^{1 / 2} P^{0}\left(\Theta_{n}\right)^{1 / 2} .
$$

For any rational $p,(4.2)$ implies that the tail of this series is dominated by $2^{-n}$. Thus $E^{0}\left(\tau^{p / 2}\right)<\infty$ for all rational $p$, and hence for all $p \in(0, \infty)$. The proof of Theorem 4.1 is complete. 
Acknowledgement. The author wishes to thank his thesis advisor, D. L. Burkholder, for his support and encouragement during the course of this work.

\section{REFERENCES}

1. A Baernstein and B. A. Taylor, Spherical rearrangements, subharmonic functions, and *-functions in n-space, Duke Math. J. 43 (1976), 245-268.

2. D. L. Burkholder, Distribution function inequalities for martingales, Ann. Probability 1 (1973), 19-42.

3. __ One sided maximal functions and $H^{p}$, J. Functional Analysis 18 (1975), 429-454.

4. Exit times of Brownian motion, harmonic majorization, and Hardy spaces, Adv. in Math. 26 (1977), 182-205.

5. Weak inequalities for exit times and analytic functions, Proceedings of the Probability Semester (Spring, 1976), Stefan Banach International Mathematical Center, Banach Center Publications, Warsaw, 1979, pp. 27-39.

6. D. L. Burkholder and R. F. Gundy, Extrapolation and interpolation of quasi-linear operators on martingales, Acta Math. 124 (1970), 249-304.

7. D. L. Burkholder, R. F. Gundy and M. L. Silverstein, A maximal function characterization of the class $H^{p}$, Trans. Amer. Math. Soc. 157 (1971), 137-153.

8. B. Davis, Brownian motion and analytic functions, Ann. Probability 7 (1979), 913-932.

9. J. L. Doob, Conditional Brownian motion and the boundary limits of harmonic functions, Bull. Math. Soc. France 85 (1957), 431-458.

10. T. R. McConnell, Inequalities for random walk and partially observed Brownian motion, Ph. D. Dissertation, University of Illinois.

11. H. P. McKean, Jr., Stochastic integrals, Probability and Math. Statist., no. 5, Academic Press, New York, 1969.

12. P. A. Meyer, Processus de Markov: le frontière de Martin, Lecture Notes in Math., vol. 77, Springer-Verlag, Berlin and New York, 1968.

13. K. Petersen, Brownian motion, Hardy spaces, and bounded mean oscillation, Cambridge Univ. Press, New York, 1977.

14. E. M. Stein, Singular integrals and differentiability properties of functions, Princeton Univ. Press, Princeton, N. J., 1970.

15. M. Tsuji, Potential theory in modern function theory, Maruzen, Tokyo, 1959.

DePartment of Mathematics, University of Illinois, Urbana, Illinois 61801 